\title{
Mechanistic determination of tear film thinning via fitting simplified models to tear breakup
}

Rayanne A. Luke ${ }^{1,2}$, Richard J. Braun ${ }^{1}$, Carolyn G. Begley ${ }^{3}$

${ }^{1}$ Department of Mathematical Sciences, University of Delaware, Newark, DE, USA, ${ }^{2}$ Current Address: Department of Applied Mathematics and Statistics, Johns Hopkins University, Baltimore, MD, USA, ${ }^{3}$ School of Optometry, Indiana University, Bloomington, IN, USA

\section{Abstract}

Purpose: Little quantitative or mechanistic information about tear film breakup can be determined directly via current imaging techniques. In this paper, we present simplified mathematical models based on two proposed mechanisms of tear film breakup: evaporation of water from the tear film and tangential fluid flow within the tear film. We use our models to determine whether one or a combination of the two mechanisms causes tear film breakup in a variety of instances. In this study, we estimate related breakup parameters that cannot currently be measured in breakup during subject trials, such as tear film osmolarity and thinning rates. The present study validates our procedure against previous work.

Methods: Five ordinary differential equation models for tear film thinning were designed that model evaporation, osmosis, and various types of tangential flow. Eight tear film breakup instances occurring within a time interval of 1-8 s postblink of five healthy subjects that were identified in fluorescence images in previous work were fit with these five models. The fitting procedure used a nonlinear least squares optimization that minimized the difference of the computed theoretical fluorescent intensity from the models and the experimental fluorescent intensity from the images. The optimization was conducted over the evaporation rate and up to three tangential flow rate parameters. The smallest norm of the difference was determined to correspond to the model that best explained the tear film dynamics.

Results: All of the breakup instances were best fit by models with time-dependent

Correspondence: Rayanne Luke, Department of Mathematical Sciences, University of Delaware, 15 Orchard Rd, Newark, DE, USA.

E-mail: rayanne@udel.edu 
tangential flow. Our optimal parameter values and thinning rate as well as tangential fluid flow profiles compare well with previous partial differential equation model results in most instances.

Conclusion: Our fitting results suggest that a combination of tangential fluid flow and evaporation cause most of the breakup instances. Comparison with results from previous work suggests that the simplified models can capture the essential tear film dynamics in most cases, thereby validating this procedure for wider usage.

Keywords: dry eye, fluorescent imaging, optimization, tear breakup, tear film

\section{Introduction}

Tear film breakup (TBU) occurs when a thinned region forms in the tear film (TF). Clinically, this is defined as the first dark area that is observed in the fluorescent (FL) TF following instillation of fluorescein dye. ${ }^{1}$ Various mechanisms are thought to cause different types of TBU: evaporation ${ }^{2-4}$ causes relatively slow thinning, ${ }^{5}$ and rapid thinning may be explained by Marangoni-driven tangential flow, ${ }^{6}$ or plausibly, dewetting in cases of circular TBU. ${ }^{7,8}$ Here, we use the phrase tangential flow to mean flow along the eye. The TF lipid layer is known to contain surface-active polar lipids, for which lipid concentration gradients induce shear stress at the aqueous/lipid interface and drive outward tangential flow by way of the Marangoni effect. ${ }^{9,10}$ Dewetting from irregularities in a corneal surface region has been hypothesized to generate outward tangential flow from internal pressure gradients inside the aqueous layer due to van der Waals type forces. ${ }^{11-13}$ A related term is full-thickness tear film breakup (full thickness-TBU), which is when the aqueous layer has thinned to the point where the lipid layer and glycocalyx touch. ${ }^{4,14}$

The effects of evaporation and the Marangoni effect on TBU have been studied extensively ${ }^{4,15}$ and modeled separately; ${ }^{16-19}$ only recently have they been explored in combination to explain breakup occurring on an intermediate time scale. ${ }^{6}$ This intermediate time scale allows us to study breakup for which lipid-driven Marangoni flow alone may be too fast, but evaporation alone is too slow to explain. Zhong et al. ${ }^{6}$ developed a partial differential equation (PDE) model with one spatial variable that incorporated both mechanisms. Luke et $a{ }^{20}$ fit full thickness-TBU data from fluorescent $(\mathrm{FL})$ images from healthy subjects with a rescaled version of the Zhong et al. ${ }^{6}$ model. The authors studied breakup occurring on the intermediate time scale of 1-8 s postblink. The optimization was conducted via nonlinear least squares minimization of the theoretical and experimental FL intensity, and TBU parameters were estimated for the model. The PDE fitting process is time-consuming and limited to spots or streaks; more complicated shapes could be fit using two spatial dimensions.

Ordinary differential equation (ODE) models have been designed to capture TF thinning while neglecting spatial variation; exact solutions exist for some cases. Braun et al. ${ }^{21}$ extended an ODE model without tangential flow from previous work ${ }^{22}$ to include time-independent extensional, tangential fluid flow; a time-dependent 
version has recently been developed and is presented here. The tangential flow is divergent from the origin and can be considered with evaporative loss and osmotic supply. Osmotic flow is directed from the corneal surface through the depth of the TF, and is perpendicular to the tangential flow. Winter et al. ${ }^{23}$ (PDE model) and Braun ${ }^{24}$ (ODE model) included van der Waals forces to stop thinning with a zero permeability condition at the tear/cornea interface; such terms and forces are omitted from the models in this work. Luke et al. ${ }^{25}$ fit TBU data with ODE models with evaporation, with or without osmosis, but without tangential flow. Neither the PDE nor ODE model gave the best fit for all of the TBU instances. The instances best fit by the ODE models had rates of $\mathrm{FL}$ intensity decrease most closely approximated by a constant.

Both TF instability and hyperosmolarity are important to study because they are proposed as etiological causes of dry eye disease (DED). ${ }^{3,26,27}$ Osmolarity is the osmotically active salt ion concentration in the aqueous layer. ${ }^{28,29} \mathrm{~A}$ concentration difference between the corneal epithelium and aqueous layer induces osmotic flow from the cornea to the TF. ${ }^{17,30}$ TF osmolarity may be measured in the inferior meniscus clinically; ${ }^{31}$ the healthy range is $296-302 \mathrm{mOsmol} / \mathrm{L} .{ }^{31-33}$ Dry eye measurements in the same location can reach $316-360 \mathrm{mOsmol} / \mathrm{L}^{26,32,34,35}$ but estimates for the TF over the cornea reach $900 \mathrm{mOsmol} / \mathrm{L}$ or higher. ${ }^{17,25,30,36}$ High levels of TF osmolarity are associated with pain, inflammation, and cellular changes. ${ }^{36-38}$ In support of these potentially high levels of TF osmolarity over the cornea, mathematical models without spatial variation have estimated peak osmolarities up to ten times the isotonic concentration. ${ }^{24,30}$ The modeling work of Peng et al. ${ }^{17}$ found that evaporation elevates osmolarity in breakup regions.

TF thinning rates have been measured experimentally or estimated in many studies. A few experimental methods include spectral interferometry, ${ }^{5,39,40}$ an open chamber, ${ }^{41}$ an evaporimeter, ${ }^{42}$ and averaging pure water and functioning lipid layer rates over the cornea obtained by heat transfer analysis and thermal imaging. ${ }^{43}$ In Braun et al., ${ }^{18}$ both peak and background evaporation rates in TBU instances, as well as the width of the evaporation distribution, were studied parametrically. Subsequently, parameter estimation schemes were developed in Luke et al. ${ }^{20,25}$ for fitting PDE models to experimental FL intensity distributions. They found evaporation rates ranging from -4.91 to $36.9 \mu \mathrm{m} / \mathrm{min}$ (the lower bound indicating thickening) and overall TF thinning rates ranging from 1.85 to $23.5 \mu \mathrm{m} / \mathrm{min}$. These thinning rates were comparable to or a little faster than previous experimental rates measured that were not specifically seeking TBU instances. ${ }^{39}$

In this paper, we fit a hierarchy of ODE models to the same dataset as in Luke et al.. ${ }^{20}$ The authors fit TBU instances with PDE models that incorporated evaporation and the Marangoni effect. ${ }^{20}$ We use these PDE results as a guide when determining whether our results have captured what we believe to be the correct dynamics. In some cases, the ODE models are better able to follow the experimental data than the PDEs, suggesting different conclusions may be drawn for those particular instances. 


\section{Methods}

\subsection{FL images}

Data was taken from 25 normal, healthy subjects in a study conducted at Indiana University, ${ }^{44}$ as discussed in previous papers. ${ }^{20,25}$ Approval was received from the Biomedical Institutional Review Board of Indiana University, Declaration of Helsinki principles were followed during data collection, and informed consent was obtained from all subjects. Subjects were seated behind a slit lamp biomicroscope and $2 \%$ sodium fluorescein solution was instilled in the patient's eye. A light with a cobalt blue excitation filter illuminated the eye so that the aqueous layer of the TF fluoresced green..$^{45} \mathrm{~A}$ trial is the sequence of images of the subject's eye following a few quick blinks. The trial records the fluorescence of the aqueous part of the TF. The trials typically start with an FL concentration close to $0.2 \%$, which is the so-called critical concentration where peak fluorescence occurs for thin TFs. ${ }^{46}$ The critical FL concentration can be expressed in molar as $0.0053 \mathrm{M}$; see Section 2.3.

We used data from five subjects out of the original 25 because for those subjects, FL intensity decrease occurred sufficiently quickly to potentially have mixedmechanism TBU. We study the same intermediate TBU data occurring in 1-8 s postblink as in Luke et al. ${ }^{20}$ in order to compare with our previous work. Many of the other 20 subjects exhibited breakup that was much slower than this intermediate time frame and thus the results in this paper may not apply to them.

For our purposes, full thickness-TBU is thinning to what is evidently a very small aqueous thickness, as determined by the aid of a computer. We fit the central data of the same extracted data from spot- or streak-shaped full thickness-TBU instances in Luke et al. ${ }^{20}$ All instances reported in this paper are shown in Figure 1 . The time resolution of our dynamic data is restricted by the frame rate, which is 4 or $5 \mathrm{~s}^{-1}$ depending on the trial.

\subsection{Governing dimensional equations}

Our single-variable ODE models are designed to capture the key ingredients in thinning in order to distinguish which is the dominant mechanism causing thinning: evaporation, outward tangential flow, or a combination of the two. Evaporationdominated thinning is characterized by inward tangential flow, if any, ${ }^{25}$ while Marangoni flow is characterized by strong outward tangential flow that decreases in strength as the trial progresses. ${ }^{20}$

There is a hierarchy of ODE models we explore; we derive the most complicated version here. This version will be referred to as the case $M$ model. Section 2.6 describes the simpler variations. We assume that evaporation, osmosis, and a combination of constant and decaying extensional flow affect the TF thickness. We use Cartesian coordinates $\left(x^{\prime}, z^{\prime}\right)$ to denote the position and $\vec{u}^{\prime}=\left(u^{\prime}, w^{\prime}\right)$ to denote the fluid velocity. Primes denote dimensional quantities. The TF is modeled as an incompressible Newtonian fluid on $0<x^{\prime}<X_{0}$ and $0<z^{\prime}<h^{\prime}\left(x^{\prime}, t^{\prime}\right)$, where $h^{\prime}\left(x^{\prime}, t^{\prime}\right)$ 




(a) S9v1t4

(b) S9v2t1

(c) $\mathrm{S} 9 \mathrm{v} 2 \mathrm{t} 5$

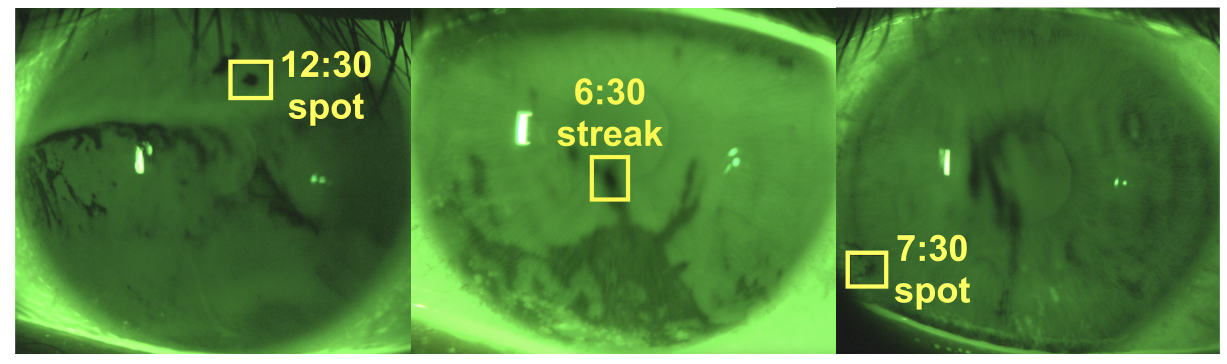

(d) S10v1t6

(e) S13v2t10

(f) S18v2t4
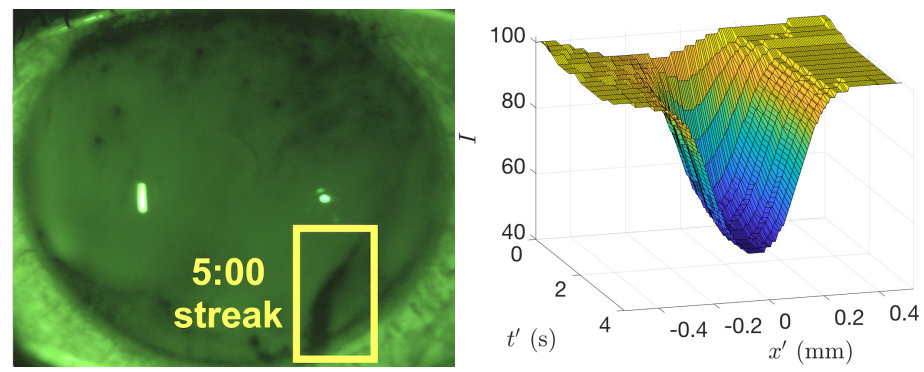

(g) S27v2t2

(h) S27v2t2 5:00 streak surface plot

Fig. 1. (a-g) The last image in each trial. The bright rectangle, called the Purkinje image, is due to the reflection from the light source. The images have been brightened for better visibility. (h) Surface plot of the FL intensity over time for subject S27v2t2 5:00 shown in (g). 
denotes the thickness of the film. Conservation of mass of TF fluid is given by:

$$
\nabla^{\prime} \cdot \vec{u}^{\prime}=0
$$

At the film/cornea interface $z^{\prime}=0$, we require osmosis across a perfect semipermeable membrane:

$$
u^{\prime}=0, \quad w^{\prime}=P_{o} V_{w}\left(c^{\prime}-c_{0}\right),
$$

where $c^{\prime}$ is the osmolarity. Osmosis is written as a concentration difference between the cornea and the TF; a greater TF osmolarity will drive osmotic flow from the cornea into the TF. The membrane permeability is given by $P_{o}$, the molar volume of water is $V_{w}$, and $c_{0}$ is the isotonic osmolarity. Dimensional parameters used in the model are summarized in Table 1. The kinematic condition at the fluid/air interface is given by:

$$
\partial_{t^{\prime}} h^{\prime}=-J^{\prime} / \rho+\left.w^{\prime}\right|_{z^{\prime}=h^{\prime}}-\left.u^{\prime}\right|_{z^{\prime}=h^{\prime}} \partial_{x^{\prime}} h^{\prime},
$$

where $J^{\prime}$ is the evaporative flux and $\rho$ is the fluid density. Since we have assumed the film is spatially uniform, we have $\partial_{x^{\prime}} h^{\prime}=0$, and thus:

$$
\dot{h^{\prime}}=-J^{\prime} / \rho+w_{z^{\prime}=h^{\prime}}^{\prime} .
$$

The dot indicates an ordinary derivative in time.

This model allows for the tangential flow to change direction: for example, it may start outward and strong, but then decay to a weakly inward, constant value. We assume a combination of constant and decaying, extensional tangential flow:

$$
u^{\prime}=\left(a^{\prime}+b_{1}^{\prime} e^{-b_{2}^{\prime} t^{\prime}}\right) x^{\prime},
$$

where $a^{\prime}$ is a constant tangential flow rate, and $b_{1}^{\prime}$ and $b_{2}^{\prime}$ are a tangential flow and decay rate, respectively. The exponential term will greatly diminish that part of the tangential flow after $1 / b_{2}^{\prime}$ units of time. An example of this fluid profile is shown in Figure 2 .

\subsection{Scalings}

As is typical for studies of this kind, we nondimensionalize the variables so the relative importance of the variables is clear. The governing equations can be nondimensionalized using the following scalings:

$c^{\prime}=c_{0} c, \quad h^{\prime}=d h, \quad t^{\prime}=(\ell / U) t, \quad u^{\prime}=U u, \quad f^{\prime}=f_{\mathrm{cr}} f, \quad J^{\prime}=\rho U J$.

Here we introduce $f^{\prime}$, the sodium fluorescein (FL) concentration. The following scalings are a result of the nondimensionalization process:

$$
w^{\prime}=\epsilon U w, \quad a^{\prime}=(U / \ell) a, \quad b_{1}^{\prime}=(U / \ell) b_{1}, \quad b_{2}^{\prime}=(U / \ell) b_{2} .
$$




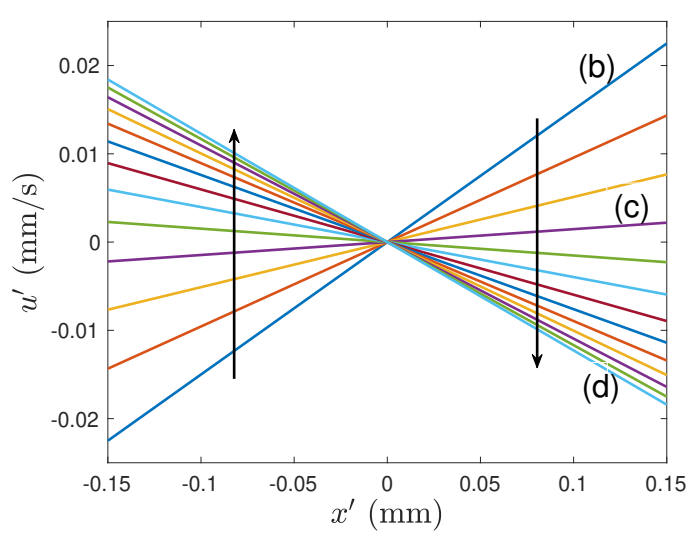

(a) Extensional, time-dependent tangential fluid flow (b)

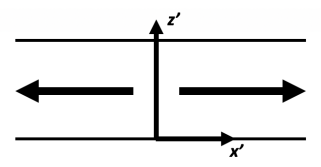

(c)



(d)

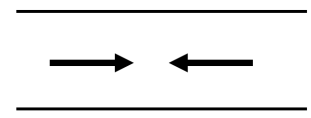

(b) Sketches of flow at (b), (c), and (d)

Fig. 2. An example of the tangential fluid flow profile $u^{\prime}$ from Equation 5. This simulation models strong outward tangential flow that dies off and then inward, capillary tangential flow persists. The parameters used are $a^{\prime}=-0.15 s^{-1}, b_{1}^{\prime}=0.3 s^{-1}$, and $b_{2}^{\prime}=0.8 s^{-1}$. A final time of $3 \mathrm{~s}$ was used. Arrows indicate increasing time.

We follow the same scalings used in Luke et al. ${ }^{20}$ to compare with their results. We take the fit interval of the trial as our time scale $t_{s}$. The characteristic horizontal fluid velocity $U$ relates the length scale $\ell$ along to the film to the time scale $t_{s}$ by $U=\ell / t_{s}$. This gives:

$$
\ell=t_{s} U=\left(\frac{t_{s} \sigma_{0} d^{3}}{\mu}\right)^{1 / 4} .
$$

The length scale along the film balances surface tension and viscosity. We have found that these length and time scales are appropriate for fitting the instances we study.

FL concentration is typically reported as a percentage in the ocular literature. For a particular FL concentration $f^{\prime}$ given as a percentage, this quantity is converted to molar as $f_{M}^{\prime}$ by:

$$
f_{M}^{\prime}=\frac{\rho}{M_{w}} \frac{f^{\prime}}{100},
$$

where $\rho$ is the density of water (Table 1$)$ and $M_{w}$ is the molecular weight of sodium fluorescein (approximately $376 \mathrm{~g} / \mathrm{mol}$ ). Critical FL concentration $f_{\mathrm{cr}}, 0.2 \%$, makes an $0.0053 \mathrm{M}$ solution when dissolved in water. This conversion of $f_{\mathrm{cr}}$ to molar is necessary to compute the dimensionless Napierian extinction coefficient $\phi$ (Table 2). 
Table 1. The dimensional parameters used are shown. The range of estimates for thinning rates are from point measurements; this range includes the values given by our optimization.

\begin{tabular}{|c|c|c|c|}
\hline \multicolumn{4}{|c|}{ Dimensional parameters } \\
\hline Parameter & Description & Value & Reference \\
\hline$\rho$ & Density & $10^{3} \mathrm{~kg} \cdot \mathrm{m}^{-3}$ & Water \\
\hline$d$ & Initial TF thickness & $2-5 \times 10^{-6} \mathrm{~m}$ & Calculated $^{20}$ \\
\hline$f_{0}^{\prime}$ & Init. FL concentration & $0.259-0.4 \%$ & Calculated $^{20}$ \\
\hline$v^{\prime}$ & $\begin{array}{l}\text { Evaporative thinning } \\
\text { rate }\end{array}$ & $0.5-25 \mu \mathrm{m} / \mathrm{min}$ & Nichols et al. ${ }^{39}$ \\
\hline$a^{\prime}$ & $\begin{array}{l}\text { Constant extensional } \\
\text { flow rate }\end{array}$ & $-0.201-1.74 s^{-1}$ & Calculated \\
\hline$b_{1}^{\prime}$ & $\begin{array}{l}\text { Decaying extensional } \\
\text { flow rate }\end{array}$ & $-0.564-3.69 s^{-1}$ & Calculated \\
\hline$b_{2}^{\prime}$ & Decay rate & $0.0421-12.8 s^{-1}$ & Calculated \\
\hline$V_{w}$ & Molar volume of water & $1.8 \times 10^{-5} \mathrm{~m}^{3} \cdot \mathrm{mol}^{-1}$ & Water \\
\hline$c_{0}$ & Isotonic osmolarity & $300 \mathrm{mOsmol} / \mathrm{L}$ & Lemp et $a l^{31}$ \\
\hline$P_{o}$ & Permeability of cornea & $12.1 \times 10^{-6} \mathrm{~m} / \mathrm{s}$ & Braun et al. $^{30}$ \\
\hline$\epsilon_{f}$ & $\begin{array}{l}\text { Naperian extinction } \\
\text { coefficient }\end{array}$ & $1.75 \times 10^{7} \mathrm{~m}^{-1} \mathrm{M}^{-1}$ & Mota et al. ${ }^{47}$ \\
\hline$\mu$ & Viscosity & $1.3 \times 10^{-3} \mathrm{~Pa} \cdot \mathrm{s}$ & Tiffany $^{48}$ \\
\hline$\sigma_{0}$ & Surface tension & $0.045 \mathrm{~N} \cdot \mathrm{m}^{-1}$ & $\begin{array}{l}\text { Nagyogá } \\
\text { Tiffany }^{49}\end{array}$ \\
\hline$\ell$ & Characteristic length & $0.138-0.412 \mathrm{~mm}$ & Calculated \\
\hline$U$ & Characteristic velocity & $0.0560-0.0990 \mathrm{~mm} / \mathrm{s}$ & Calculated \\
\hline$t_{s}$ & Time scale & $1.75-6.6 \mathrm{~s}$ & Fit interval $^{20}$ \\
\hline
\end{tabular}

\subsection{Derivation of TF equations}

Using the scalings of Equations 6 and 7, we nondimensionalize the governing equations. From the nondimensional version of Equation 5, the strain rate is:

$$
\partial_{x} u=a+b_{1} e^{-b_{2} t} .
$$

In Cartesian coordinates, conservation of mass is given by:

$$
\partial_{x} u+\partial_{z} w=0 .
$$

We replace $\partial_{x} u$ in Equation 11 using Equation 10, integrate Equation 11 over the vertical domain, and then replace $w_{z=h}$ using Equation 4. This gives:

$$
0=\int_{0}^{h}\left[\partial_{x} u+\partial_{z} w\right] d z=\partial_{t} h+J-P_{c}(c-1)-\left(a+b_{1} e^{-b_{2} t}\right) h,
$$

where we have used the independence of $\partial_{x} u$ from $z$. Here, the nondimensional evaporation rate is $J$, and $P_{c}$ is the nondimensional permeability constant. Rewrit- 
Table 2. Dimensionless parameters that arise from scaling the dimensional fluid mechanics problem. The values given are based upon the values of Table 1 and those used to generate Figure 4.

\begin{tabular}{|l|l|l|l|}
\hline \multicolumn{4}{|c|}{ Nondimensional parameters with typical values } \\
\hline Parameter & Description & Expression & Value \\
\hline$\epsilon$ & Aspect ratio & $d / \ell$ & 0.0130 \\
$P_{c}$ & Permeability of cornea & $P_{o} V_{w} c_{0} /(\epsilon U)$ & 0.0653 \\
$\phi$ & Nondimensional Napierian & $\epsilon_{f} f_{\text {cr }} d$ & 0.279 \\
& extinction coefficient & & \\
\hline
\end{tabular}

ing this result as a differential equation for $h$, we have:

$$
\dot{h}=-J+P_{c}(c-1)-\left(a+b_{1} e^{-b_{2} t}\right) h .
$$

Our transport equation for solute $s=c$ or $f$ is (without any spatial terms):

$$
h \dot{s}=J s-P_{c}(c-1) s .
$$

Multiplying Equation 13 by $s$ and adding the result to Equation 14, we have an ODE for the product $h s$ :

$$
(\dot{h} s)=-\left(a+b_{1} e^{-b_{2} t}\right) h s .
$$

For this and all versions of the model, solute conservation relates the derivative of the solute mass $(h s)$ to the strain rate. Separating and integrating gives:

$$
h s=A \exp \left(-a t+\frac{b_{1}}{b_{2}} e^{-b t}\right)
$$

where $A$ is an arbitrary constant of integration. The constant $A$ will be found for $s=c$ or $f$ in Section 2.5.

\subsection{Initial conditions}

At the start of a trial, sodium fluorescein is instilled, and after a few blinks we can estimate both fluorescein concentration and TF thickness, as will be discussed in Section 2.8. We assume isotonic osmolarity for our starting concentration. Using our scalings in Equation 6, our initial conditions are normalized based on these initial estimated values. In all cases of the model, we have uniform nondimensional initial conditions:

$$
c(0)=1, h(0)=1 \text {, and } f(0)=f_{0} .
$$

Using Equation 17, we solve Equation 16 for $c$ and $f$ separately:

$$
c=\frac{1}{h} \exp \left[-a t+\frac{b_{1}}{b_{2}}\left(e^{-b_{2} t}-1\right)\right],
$$




$$
f=\frac{f_{0}}{h} \exp \left[-a t+\frac{b_{1}}{b_{2}}\left(e^{-b_{2} t}-1\right)\right] .
$$

Substituting for $c$ in Equation 13 via Equation 18 leads to our equation for nondimensional film thickness, $h(t)$, governed by:

$$
\dot{h}=-J+P_{c}\left\{\frac{1}{h} \exp \left[-a t+\frac{b_{1}}{b_{2}}\left(e^{-b_{2} t}-1\right)\right]-1\right\}-\left(a+b_{1} e^{-b_{2} t}\right) h .
$$

Thus, the only initial condition needed is the one for $h$, and $c$ or $f$ may be calculated post-facto via Equation 18 and Equation 19.

\subsection{Models}

The most complicated ODE model we explore, referred to as case M, is derived in Section 2.4. The case $\mathrm{M}$ model is the most complicated as it allows for the most complex tangential fluid flow we study in this paper. We now present the simpler variations. Each variation of the model is determined by which mechanisms are assumed to affect the TF. The options are a combination of evaporation, osmosis, and tangential flow. If tangential flow is present, there are several choices we use. The simpler versions either have less complex tangential flow profiles than model $\mathrm{M}$ or ignore tangential flow and/or osmosis entirely. Each of the following variations can be obtained from case $\mathrm{M}$ by setting parameters in the case $\mathrm{M}$ model to zero.

\subsubsection{Case E model}

The simplest variation of the model assumes that constant evaporation is the only mechanism affecting the TF thickness. The differential equation can be obtained from the case $\mathrm{M}$ model by setting $P_{c}=a=b_{1}=0$ in Equation 13, which effectively shuts off osmosis and tangential flow. The case $\mathrm{E}$ differential equation is given by:

$$
\dot{h}=-J \text {. }
$$

\subsubsection{Case O model}

In this model, we add osmosis, so that we assume constant evaporation and osmosis affect the TF thickness. Figure 3 shows a sketch of the model (on left). Setting $a=b_{1}=0$ in Equation 13 turns off tangential flow in the case $\mathrm{M}$ model to give the differential equation for case 0 :

$$
\dot{h}=-J+P_{c}(c-1) .
$$

As in Section 2.5, mass conservation of solute (osmolarity), namely, $h c=1$, allows us to eliminate $c$ as in Braun et al. ${ }^{22}$ to obtain a single ODE for $h$ :

$$
\dot{h}=-J+P_{c}\left[\frac{1}{h}-1\right] \text {. }
$$


Cases $\mathrm{E}$ and $\mathrm{O}$ have zero tangential flow in order to model situations where evaporation is the dominant mechanism affecting TF thinning and tangential flow is not important.


Fig. 3. Schematic for the case $\mathrm{O}$ and F models. In contrast to case $\mathrm{M}$, neither model has timedependent tangential flow.

\subsubsection{Case F model}

In the case $\mathrm{F}$ model, we add steady extensional tangential flow to the case $\mathrm{O}$ assumptions. Thus, we assume that evaporation, osmosis, and steady, extensional tangential flow affect the thickness of the TF. Figure 3 shows a sketch of this model (on right). Setting $b_{1}=0$ in the case M model ( Equation 13) shuts off time-dependent tangential flow and results in the flow profile:

$$
u(x, t)=u(x)=a x,
$$

where $\partial_{x} u=a$ is the strain rate. The sign of $a$ determines the kind of tangential flow present. If $a<0$, the tangential flow is inward, mimicking healing, capillary tangential flow. This characterizes evaporation-dominated thinning. If $a>0$, the tangential flow is outward, mimicking Marangoni flow, driven by interfacial shear stress, and may be thought of as stretching taffy. A single curve of Figure 2 illustrates this tangential flow profile. Mass conservation becomes $h c=e^{-a t}$, and the differential equation for the TF thickness is:

$$
\dot{h}=-J+P_{c}\left[\frac{\exp (-a t)}{h}-1\right]-a h .
$$

\subsubsection{Case D model}

This model is designed to mimic the time-dependent tangential flow seen in TBU instances where tangential flow is dominated by the Marangoni effect. We assume evaporation, osmosis, and decaying, extensional tangential flow affect the TF thickness. The differential equation can be obtained from case $M$ by setting $a=0$ in (13). Here:

$$
u(x, t)=b_{1} e^{-b_{2} t} x .
$$


In this case, the strain rate is $\partial_{x} u=b_{1} e^{-b_{2} t}$. The differential equation for TF thickness $h$ is:

$$
\dot{h}=-J+P_{c}\left\{\frac{1}{h} \exp \left[\frac{b_{1}}{b_{2}}\left(e^{-b_{2} t}-1\right)\right]-1\right\}-b_{1} e^{-b_{2} t} h,
$$

where the first term inside the brackets is the result of using mass conservation to eliminate $c$.

\subsection{FL intensity}

The equation for FL intensity calculated by dimensional quantities is:

$$
I=I_{0} \frac{1-e^{-\epsilon_{f} h^{\prime} f^{\prime}}}{\left(f_{\mathrm{cr}}^{\prime}\right)^{2}+\left(f^{\prime}\right)^{2}},
$$

where $\epsilon_{f}$ is the molar Napierian extinction coefficient, $f_{\mathrm{cr}}^{\prime}$ is the critical fluorescein concentration, and $I_{0}$ is a normalization factor. Using the scalings in Equation 6, nondimensional FL intensity $I$ from the TF is given by:

$$
I=I_{0} \frac{1-\exp (-\phi h f)}{\left(1+f^{2}\right)} .
$$

Just as $c$ was replaced in Equation 13 to arrive at Equation 20, $f$ can be eliminated in Equation 29 via Equation 19 so that the $\mathrm{FL}$ intensity $I$ for the case $\mathrm{M}$ model is given by:

$$
I=I_{0} \frac{1-\exp \left\{-\phi f_{0} \exp \left[-a t+\frac{b_{1}}{b_{2}}\left(e^{-b_{2} t}-1\right)\right]\right\}}{1+\left\{f_{0} \exp \left[-a t+\frac{b_{1}}{b_{2}}\left(e^{-b_{2} t}-1\right)\right] / h\right\}^{2}} .
$$

The expressions for $I$ for the other cases can be obtained by setting $b_{1}=0$ if there is no time-dependence in the tangential flow, by setting $a=0$ if there is only timedependent tangential flow, and by setting $a=b_{1}=0$ if there is no tangential flow at all.

\subsection{Estimating initial physical quantities}

We estimate the initial FL concentration following Wu et $a{ }^{50}$. This value is assumed to be uniform throughout the TF. By inverting Equation 28 for $h^{\prime}$, we obtain an initial TF thickness estimate:

$$
h_{0}^{\prime}=-\frac{1}{\epsilon_{f} f_{0}^{\prime}} \log \left\{1-\frac{I}{I_{0}}\left[1+\left(\frac{f_{0}^{\prime}}{f_{\mathrm{cr}}}\right)^{2}\right]\right\} .
$$

Model eye calculations ${ }^{50}$ determine $I_{0}$ through a least squares problem. The relative initial FL intensity $I$ at the center of the TBU instance, where the minimum FL intensity in the corneal region of interest has been subtracted off, is used. More details about the procedure can be found in Luke et al. ${ }^{25}$ 


\subsection{Optimization}

We follow the process described in Luke et al.;25 a summary is given below.

\subsubsection{Data preparation}

We use custom MATLAB codes to convert the images in each trial from RGB color to grayscale, smooth the images with a Gaussian filter, and stabilize the images using the Purkinje image, ${ }^{44}$ a bright artefact reflecting the light source. We use the same roughly linear or circular full thickness-TBU instances that were chosen and fit by a PDE model in Luke et al. ${ }^{20}$ to compare with the PDE results. We fit our theoretical FL intensity to the central data of a subset of approximately 6-10 time levels of experimental FL intensity data from the trial. The starting frame is the last frame before the FL intensity data starts decreasing. The first few frames of a trial are taken at a lower light setting to obtain an initial FL concentration estimate, and in some trials there is evidence that thinning has begun during this interval. As a result, the first bright image already exhibits significant decrease in FL intensity in the center of breakup. Luke et $a l .{ }^{20}$ remedied this issue by introducing "ghost" time levels, allowing the model solution to start with a uniform time level that is not compared to the experimental FL data. This is a product of the low time resolution of our data. In this work, we also use "ghost" times as appropriate. The last frame is the final frame before the FL intensity data stop decreasing.

\subsubsection{Optimization problem}

We discuss the optimization for the case $\mathrm{M}$ model. Expressed in continuous variables, we seek to minimize $\left\|I_{\mathrm{th}}(t)-I_{\mathrm{ex}}(t)\right\|_{2}^{2}$ over the parameters $v^{\prime}$, the evaporation rate, $a^{\prime}$, the constant extensional tangential flow rate, $b_{1}^{\prime}$, the decaying extensional tangential flow rate, and $b_{2}^{\prime}$, the decay rate. Here, $t$ corresponds to the time after the light source brightness has been increased to the high setting. The norm is over all $t \in[0, T]$ excluding any "ghost" time levels from the theoretical FL intensity, where $T$ corresponds to the total length of the trial. The optimization problem may be written:

$$
\underset{v^{\prime}, a^{\prime}, b_{1}^{\prime}, b_{2}^{\prime}}{\operatorname{argmin}}\left\|I_{\mathrm{th}}\left(t ; v^{\prime}, a^{\prime}, b_{1}^{\prime}, b_{2}^{\prime}\right)-I_{\mathrm{ex}}(t)\right\|_{2}^{2} .
$$

Theoretical intensity, $I_{\text {th }}$, is computed after solving the differential equation for film thickness, $h$. Similar optimizations are conducted for each of the other models.

\subsubsection{Numerical method and stopping criterion}

The five ODEs for $h$ are solved using ode15s in MATLAB (MathWorks, Natick, MA, USA). For the optimization, we use a nonlinear least squares minimization implemented by Isqnonlin in MATLAB with the trust-region reflective algorithm ${ }^{51}$ and we add a second order finite difference approximation of the Jacobian ${ }^{52}$ to improve performance. To 
generate initial guesses for optimization, forward computations were conducted until the theoretical dynamics were close to the experimental. For each instance, the solver stopped because the change in residual was less than the specified tolerance. Optimization tolerances of roughly the square root of the solver tolerances were used.

\section{Results}

\subsection{Exact solutions}

Exact solutions exist for the case $\mathrm{E}$ model and for case $\mathrm{F}$ with $J=P_{c}=0$. For the case $\mathrm{E}$ model, using our initial condition, the nondimensional exact solution is:

$$
h(t)=1-J t .
$$

This solution ignores the physical reality that evaporation ceases once the TF reaches zero thickness; thus, the solution is only relevant for $t \in[0,1 / J]$.

If we assume that time-independent tangential flow is the only mechanism affecting the TF thickness in case $\mathrm{F}$, then $J=P_{c}=0$ in (6) applies:

$$
\dot{h}=-a h \text {. }
$$

Using our initial condition, we find that:

$$
h(t)=e^{-a t} .
$$

To model TF thinning, we assume $a>0$ in this instance. Thus, as expected, the TF thins to zero as time increases. ${ }^{53}$

\subsection{Numerical solutions}

We now discuss example solutions in nondimensional form for all cases of the model. In Figure 4 we plot these nondimensional theoretical solutions. For comparison purposes, we have used the same parameter values for each of the five models. In particular, both tangential flow parameters are positive, indicating outward tangential flow. The nondimensional parameters that result from our scalings are $a=0.45, b_{1}=$ $0.9, b_{2}=2.4, J=0.5$, and $P_{c}=0.0653$. We see that the case $O$ solution thins slightly less than the case $E$ solution due to osmosis, which adds fluid to the TF. For the three models involving tangential flow, since we have selected both $a, b_{1}>0$, the case $\mathrm{M}$ model shows the most thinning since the outward tangential flow is the strongest among all the models. The case F and D model solutions for $h$ are similar early on since they differ only by their tangential flow profiles. At later times, the case $D$ tangential flow shuts off, causing the solution to level off. In contrast, the case $\mathrm{F}$ tangential flow is constant and persists at later times, causing the case $\mathrm{F}$ solution to thin more than case D. Osmolarity and normalized FL concentration solutions are identical in the absence of spatial variation. Both quantities are inversely related to 
TF thickness and increase at the origin in the presence of outward tangential flow; the profiles reach the highest peak value for the case $\mathrm{M}$ model, which exhibits the greatest decrease in TF thickness and the strongest tangential fluid flow.

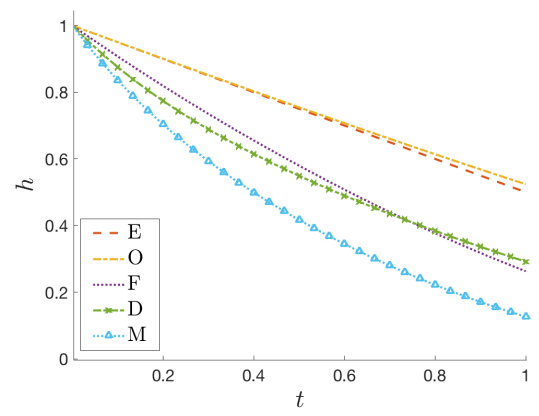

(a) TF thickness

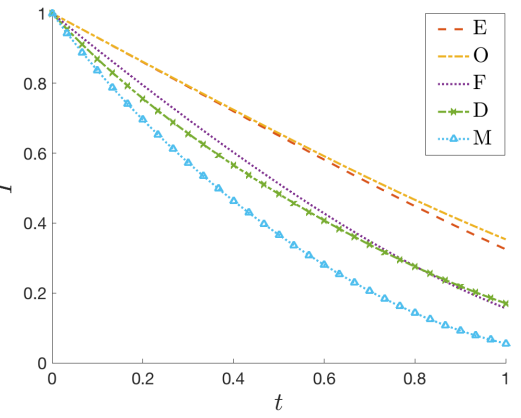

(b) FL intensity

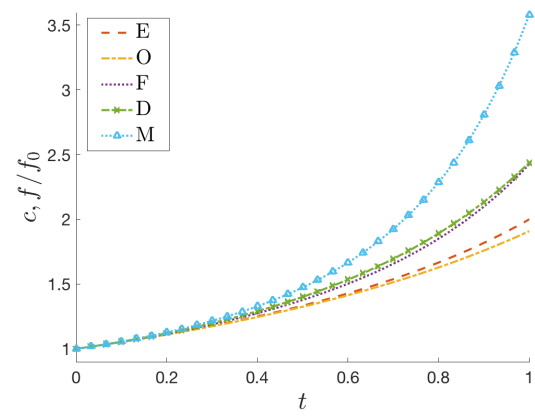

(c) Normalized solute concentration

Fig. 4. Nondimensional theoretical solutions for the five cases of the model with $v^{\prime}=30 \mu \mathrm{m} / \mathrm{min}, a^{\prime}=0.15 / \mathrm{s}, b_{1}^{\prime}=0.3 / \mathrm{s}, b_{2}^{\prime}=0.8 / \mathrm{s}, f_{0}^{\prime}=0.3 \%, d=3 \mu \mathrm{m}$, and $t_{s}=3 \mathrm{~s}$.

\subsection{Fitting examples}

We fit the same instances that were fit with the mixed-mechanism PDE model of Luke et al.; ${ }^{20}$ we present a few examples. The S10v1t6 12:30 spot is shown as an example of our full fitting procedure in Figure 5. Figure $5 c$ shows the line of data extracted for the PDE fit recorded in Luke et al. ${ }^{20}$ we fit the breakup data at the midpoint of the line with our ODE models. The results for each of the five ODE models are recorded in Table 3 and compared with the corresponding PDE model result.

The first $6 \mathrm{~s}$ of the trial are obscured by eyelashes and the upper eyelid. The spot has already started to form and darkens quickly after the breakup region is revealed around $6 \mathrm{~s}$ into the trial (see Fig. 5b). In order to fit the data with our model, we use 


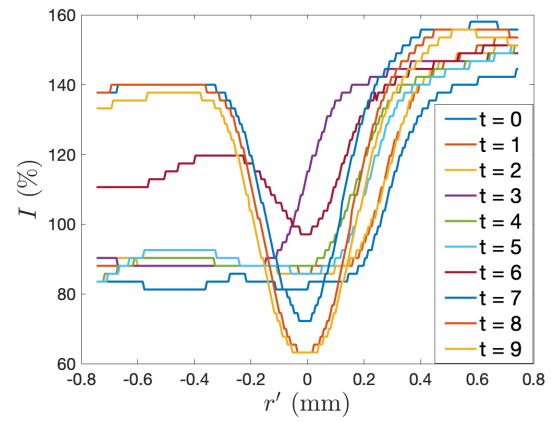

(a) FL intensity with minima aligned

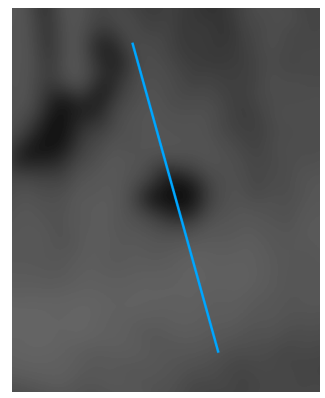

(c) Full thickness-TBU data extraction

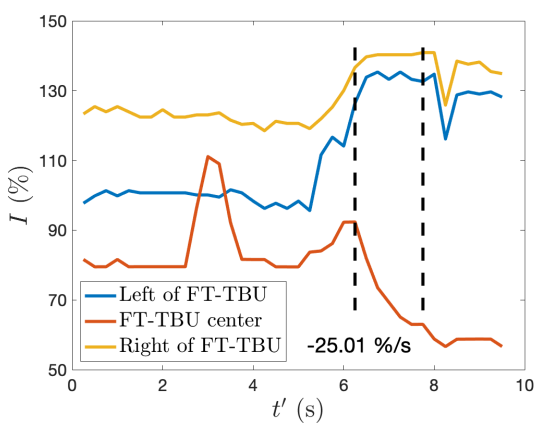

(b) FL intensity decrease

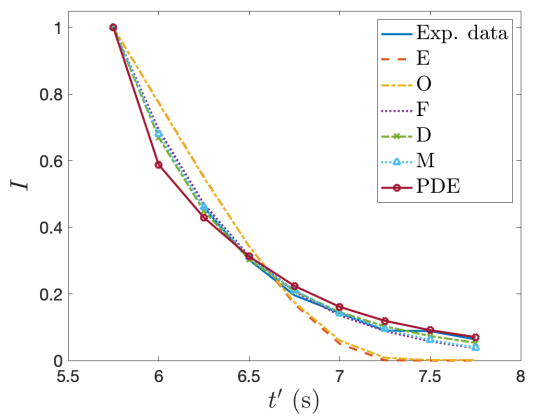

(d) Experimental and best fit theoretical FL intensity

Fig. 5. Extracted data and best fit results for the S10v1t6 12:30 spot. In (c), the image has been brightened and contrast-enhanced. Case (c) evaporation (see Luke et $a{ }^{20}{ }^{20}$ ) was used in the PDE fit. Figure (a-c) is reprinted from Luke et al. $^{20}$ with the kind permission of Springer Nature.

"ghost" time levels for $0.5 \mathrm{~s}$. Figure $5 \mathrm{~d}$ shows that the experimental FL intensity drops to less than $10 \%$ of its initial value.

In Table 3, the two ODE models without tangential flow select unrealistic evaporation rates in an attempt to match the rapid thinning of the S10v1t6 12:30 spot. On average, the evaporation rates chosen by the ODE models with tangential flow are among the smallest optimal values for all mixed-mechanism fit instances. This is likely due to the relatively large tangential flow rate parameters; unlike any other trial, the initial tangential flow value $b_{1}^{\prime}$ or $a^{\prime}$ is above $1 \mathrm{~s}^{-1}$ for each of the three models that involve tangential flow. We take this as strong evidence that the Marangoni effect is the dominant mechanism causing the thinning. Further evidence of this statement is the fact that the case $\mathrm{F}$ model selected zero evaporation. This instance was fit well with a Marangoni effect-only PDE model that ignored evaporation, which is consistent with our small or zero optimal evaporation rates. The case D model produces 
the smallest residual. This full thickness-TBU instance exhibits the largest drop in FL intensity of all eight; the decaying model gives the best fit because the TF has likely thinned to almost zero thickness, allowing little tangential flow to persist, if any.

Table 3. S10v1t6 12:30 center of spot data fit with the five cases of models. The model giving the smallest norm is shaded. The central data of the best PDE fit is shown for comparison.

\begin{tabular}{|l|l|l|l|l|l|l|}
\hline Model & $\begin{array}{c}\boldsymbol{v}^{\prime} \\
\left(\frac{\boldsymbol{\mu}}{\mathrm{min}}\right)\end{array}$ & $\begin{array}{c}\boldsymbol{a}^{\prime} \\
\left(\boldsymbol{s}^{-\mathbf{1}}\right)\end{array}$ & $\begin{array}{c}\boldsymbol{b}_{\mathbf{1}}^{\prime} \\
\left(\mathbf{s}^{-\mathbf{1}}\right)\end{array}$ & $\begin{array}{c}\boldsymbol{b}_{\mathbf{2}}^{\prime} \\
\left(\boldsymbol{s}^{-\mathbf{1}}\right)\end{array}$ & Residual & Norm \\
\hline Evap only (E) & 120 & & & & $3.88 \times 10^{-2}$ & $1.97 \times 10^{-1}$ \\
\hline Evap + osm (O) & 122 & & & & $3.47 \times 10^{-2}$ & $1.86 \times 10^{-1}$ \\
\hline $\begin{array}{c}\text { Evap, osm, } \\
\text { flow (F) }\end{array}$ & 0.00 & 1.74 & & & $2.0 \times 10^{-3}$ & $4.50 \times 10^{-2}$ \\
\hline $\begin{array}{c}\text { Evap, osm, } \\
\text { dec. flow (D) }\end{array}$ & 1.27 & & 1.95 & 0.277 & $7.86 \times 10^{-4}$ & $2.80 \times 10^{-2}$ \\
\hline $\begin{array}{c}\text { Evap, osm, } \\
\text { mixed flow (M) }\end{array}$ & 4.91 & 0.656 & 1.19 & 0.423 & $6.10 \times 10^{-4}$ & $4.04 \times 10^{-2}$ \\
\hline $\begin{array}{c}\text { Mixed-mech } \\
\text { PDE center (M) }\end{array}$ & 5.92 & & & & & $5.58 \times 10^{-2}$ \\
\hline
\end{tabular}

The S27v2t2 5:00 streak data and fits are shown in Figure 6. As in Luke et al. ${ }^{20}$, we use a quarter second of "ghost" time at the start of the fit. This instance is of particular interest because the center of the mixed-mechanism PDE theoretical FL intensity does not capture the dynamics of the experimental data well. In Luke et al. ${ }^{20}$, the S27v2t2 5:00 streak was categorized as Marangoni-effect dominated due to the large Marangoni number and outward tangential flow of the best fit. The best fit case $M$ model selects outward tangential flow; however, the close second-best (D) and thirdbest (F) cases select inward tangential flow. These latter two models also select a significantly larger evaporation rate than the others. The S27v2t2 5:00 streak was also fit with an evaporation-only mode ${ }^{18}$ in Luke et al. ${ }^{20}$. That fit (E PDE) is shown along with the mixed-mechanism fit (MM PDE) and the ODE results in Figure $6 \mathrm{~b}$ and outperforms the mixed-mechanism PDE fit. Further, the optimal peak evaporation rate for the evaporation-only PDE fit is $35.3 \mu \mathrm{m} / \mathrm{min}$, which is a large but plausible evaporation rate. This suggests that evaporation may play a larger role in this instance than previously thought.

In Figure 7a we show the S9v2t5 4:00 spot data and in Figure 7b we show the fits. We have plotted the central data from both the best-fit mixed-mechanism (MM PDE) and evaporation-only (E PDE) models for comparison because this instance is also fit well with the latter model and was categorized as evaporation-dominated in Luke et $a .^{20}$. All three ODE models with tangential flow select some amount of inward tangential flow, which aligns well with the PDE model, whose tangential flow profile changes sign at the origin as time progresses (see Table 6). In all cases, the tangential flow is of a significantly smaller magnitude than the Marangoni effect-dominated or transitional thinning instances. The case $\mathrm{M}$ model gives the smallest residual. No- 


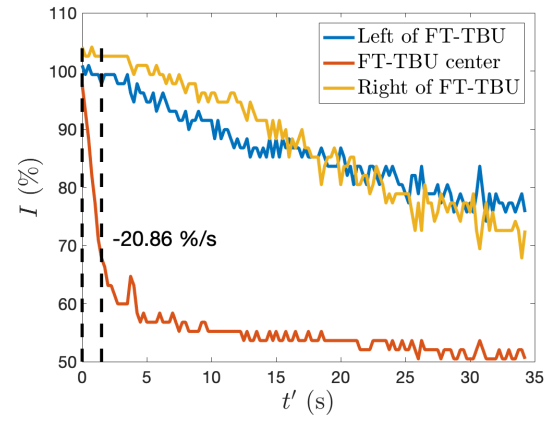

(a) FL intensity decrease



(b) Experimental and best fit theoretical FL intensity

Fig. 6. Extracted data and best fit results for the S27v2t2 5:00 streak. Uniform evaporation was used in the mixed-mechanism PDE fit.

tably, the evaporation-only fits for this instance give closer residuals to the best fit model than other instances; this suggests that evaporation is the dominant mechanism causing the thinning.



(a) FL intensity decrease

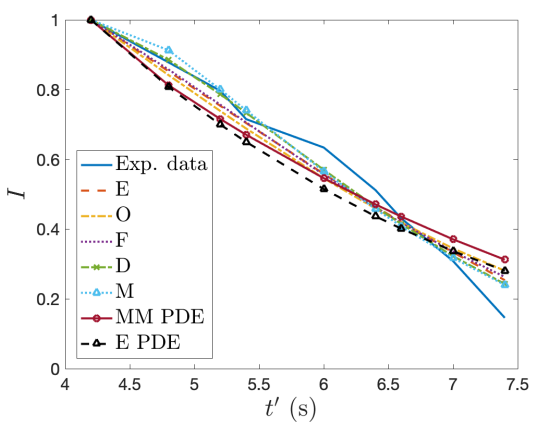

(b) Experimental and best fit theoretical FL intensity

Fig. 7. Extracted data and best fit results for the S9v2t5 4:00 spot data. Case (c) evaporation (see Luke et $a l^{20}$ ) was used in the mixed-mechanism PDE fit. Figure $(a)$ is reprinted from Luke et $a l .{ }^{20}$ with the kind permission of Springer Nature.

\subsection{Aggregate fitting results}

We now present our aggregate results of fitting the same instances that were fit with the mixed-mechanism PDE model of Luke et $a .^{20}$ In order to determine the model selected to report for each full thickness-TBU instance, a table is used to compare the 2norms of the difference between the theoretical and experimental FL intensities and we select the case of the model corresponding to the smallest value (see Table 3 for 
an example). These best fit results as determined by the smallest norm are shown in Table 4. Each full thickness-TBU instance is labeled by subject (S), visit (v), and trial $(t)$ number. The full thickness-TBU identification (FT-TBU ID) is given as a location and type as follows: the location is given as a clock reading, and the type is either a streak or spot. For example, the entries in the first row and first two columns of Table 4 should be interpreted as such: Subject 9, visit 1, trial 4; a "ghost" time level was used in the PDE fit; extracted data from the 4:00 streak as measured as a clock reading taken from the center of the pupil (inferior temporal region, see Fig. 1a). Images showing the full thickness-TBU instances can be found in Section 2.1. A combination of the evaporation rate, constant tangential flow rate, decaying tangential flow rate, and decay rate are adjusted to accomplish the fit. The optimal parameters are given for the case of the model with the smallest norm. Section 3.3 shows examples of the experimental data, fits, and resulting theoretical solutions using the optimal parameters found by nonlinear least squares minimization. The S18v2t4 7:30 spot was originally fit with a single "ghost" time level in Luke et al. ${ }^{20}$ but alternatively fit with two in the supplementary material; we choose to fit with two here as well.

Table 4. Results from fitting various ODE models (up to four parameters). The subject number (S), visit number $(v)$ and trial number $(t)$ are listed. A + denotes using a "ghost" first time level in the PDE fit and "ghost" time in the ODE fit. The full thickness-TBU location a clock reading taken from the center of the pupil. Full thickness-TBU type is denoted by a dash (-) for a streak, and a circle (o) for a spot. The initial TF thickness and FL concentration estimates are given. The optimal parameters are given for the case of the model with the smallest norm. The evaporative thinning rates are given by $v^{\prime}$, constant, extensional tangential flow rates by $a^{\prime}$ and decaying extensional tangential flow and decay rates by $b_{1}^{\prime}$ and $b_{2}^{\prime}$.

\begin{tabular}{|l|l|l|l|l|l|l|l|l|l|}
\hline Trial & $\begin{array}{c}\text { FT-TBU } \\
\text { ID }\end{array}$ & $\begin{array}{c}\boldsymbol{h}_{\mathbf{0}}^{\prime} \\
(\boldsymbol{\mu} \boldsymbol{m})\end{array}$ & $\begin{array}{c}\boldsymbol{f}_{\mathbf{0}}^{\prime} \\
(\%)\end{array}$ & $\begin{array}{c}\boldsymbol{v}^{\prime} \\
\left(\frac{\boldsymbol{\mu m}}{\mathrm{min}}\right)\end{array}$ & $\begin{array}{c}\boldsymbol{a}^{\prime} \\
\left(s^{-\mathbf{1}}\right)\end{array}$ & $\begin{array}{c}\boldsymbol{b}_{\mathbf{1}}^{\prime} \\
\left(\boldsymbol{s}^{-\mathbf{1}}\right)\end{array}$ & $\begin{array}{c}\boldsymbol{b}_{\mathbf{2}}^{\prime} \\
\left(\boldsymbol{s}^{-\mathbf{1}}\right)\end{array}$ & Norm & Model \\
\hline S9v1t4 & $4: 00-$ & 3.32 & .324 & 24.1 & .0316 & .418 & 5.75 & .203 & $\mathrm{M}$ \\
\hline S9v2t1 & $3: 00-$ & 5.01 & .292 & 27.3 & .461 & -.490 & .0715 & .110 & $\mathrm{M}$ \\
\hline S9v2t5 & $4: 00 \circ$ & 2.1 & .299 & 22.4 & .217 & -.417 & .882 & .118 & $\mathrm{M}$ \\
\hline S9v2t5 & $4: 30 \circ$ & 2.33 & .299 & 50.9 & .360 & -.564 & .367 & .192 & $\mathrm{M}$ \\
\hline S10v1t6 + & $12: 30 \circ$ & 3.08 & .293 & 1.27 & & 1.95 & .277 & .0280 & $\mathrm{D}$ \\
\hline S13v2t10+ & $6: 30-$ & 3.59 & .259 & 26.4 & & .138 & .102 & .121 & $\mathrm{D}$ \\
\hline S18v2t4 & $7: 30 \circ$ & 2.48 & .363 & 25.2 & & 2.41 & 8.85 & .111 & $\mathrm{D}$ \\
\hline S27v2t2 & $5: 00-$ & 1.91 & .4 & 9.32 & .714 & -.368 & .540 & .0271 & $\mathrm{M}$ \\
\hline
\end{tabular}

Five of the full thickness-TBU instances are best fit by the case M model and the other three are best fit by the case D model; recall that these two versions are specifically designed to capture time-dependent tangential flow that mimics the Marangoni effect. Notably, the versions of the model without tangential flow produce theoretical solutions with worse fits in all eight instances; we take this as strong evidence that tangential flow plays a crucial role in causing the TF thinning. It is worth mentioning a few other fits: the S27v2t2 5:00 streak is also fit well with the case D model, with the case $\mathrm{F}$ model not far behind; the case M model fits the S18v2t4 7:30 spot data well; and the S13v2t10 6:30 spot data also matches reasonably well with the case F model. 
Some instances exhibit more Marangoni flow than others; this is indicated by large, positive tangential flow rate parameters $a^{\prime}$ and/or $b_{1}^{\prime}$ in Table 4. The S18v2t4 7:30 and S10v1t6 12:30 spots have the strongest outward tangential flow of all instances, with initial strain rates close to or over $2 s^{-1}$. Further, all five TBU instances labeled in Luke et al. ${ }^{20}$ as "Marangoni effect-dominated" exhibit outward tangential flow, the characteristic direction of Marangoni flow. The S9v2t1 3:00 streak and S9v2t5 4:00 and 4:30 spots are designated as "evaporation-dominated" or "transitional thinning" in the PDE paper; ${ }^{20}$ these all show some amount of inward tangential flow, which is characteristic of evaporation-dominated thinning. Each of the eight instances have some amount of outward tangential flow and a nonzero evaporation rate, providing strong evidence that the Marangoni effect and evaporation act in combination to cause the TBU. This mixed-mechanism designation is consistent with our previous PDE results. ${ }^{20}$

Table 4 shows a wide range of evaporation rates, indicating the relative importance of evaporation in each TBU instance. Notably, the S9v2t5 4:30 spot instance, which was categorized as evaporation-dominated in Luke et al. ${ }^{20}$ has the highest optimal evaporation rate. In contrast, the S10v1t6 12:30 spot and S27v2t2 5:00 streak are faster instances that were categorized as Marangoni effect-dominated in the aforementioned paper; these cases exhibit the two smallest evaporation rates seen in Table 4. Corresponding osmolarities are reported in the Discussion (see Figure 11a).

\section{Discussion}

The quantities recorded in Table 4 show more variation than the PDE results in some cases, but the qualitative similarities in the solutions are an important takeaway. For each TBU instance, the best fit ODE model includes time-dependent tangential flow. This is strong evidence that evaporation alone cannot explain this thinning and that the Marangoni effect played a role, since it is characterized by nonconstant thinning.

In Figure 8 we show the various time derivatives $\dot{h}^{\prime}$ computed from the optimal values of the ODE models as well as the optimal $\partial_{t^{\prime}} h^{\prime}$ measured at the origin for the three examples of mixed-mechanism fitting shown in Section 3.3. The average starting $2 \mathrm{~s}$ in or the value at the final time point is recorded in Table 5. This delay in averaging matches the approach in Luke et al. ${ }^{20}$ and mimics experimental procedures. ${ }^{39}$ These values are shown along with the optimal evaporation rates for comparison. The S10v1t6 12:30 spot, which showed the most rapid thinning when fit with the mixedmechanism PDE model, shows dynamic rates of thinning for a large portion of the trial for many of the ODE fits in Figure 8a. The case (D) and (M) ODE model $\dot{h}^{\prime}$ values are very close, which we expect since they gave similar residuals when fit to the data. The S9v2t5 4:00 spot shows nonconstant dynamics near the end of the trial in Figure $8 \mathrm{c}$ and we see further qualitative and quantitative agreement between the mixedmechanism and evaporation-only PDE results. The case (D) and (M) models for this instance also show $\dot{h}^{\prime}>0$ in the first quarter second; the theoretical TF thickness so- 
lution is in fact slightly positive early on. This is likely an attempt by the optimization to fit the concave down portion of the data in the first second or so. In general, the PDE models produce $\partial_{t^{\prime}} h^{\prime}$ values in the first quarter second that are much larger than the corresponding ODE numbers.

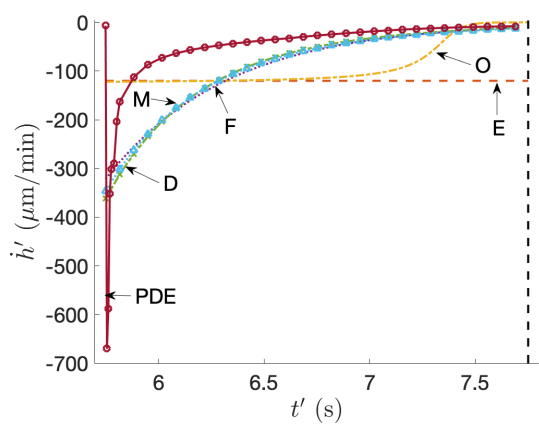

(a) S10v1t6 12:30 spot

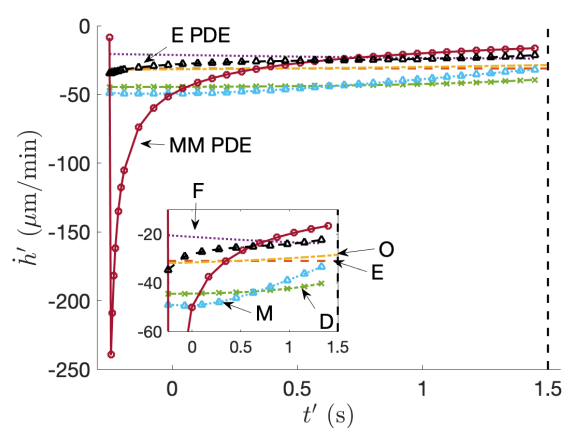

(b) S27v2t2 5:00 streak



(c) S9v2t5 4:00 spot

Fig. 8. $\dot{h}^{\prime}$ from the five ODE models are plotted alongside $\partial_{t^{\prime}} h^{\prime}$ from the PDE model. The time point at which averaging will begin is shown as a dashed vertical line.

In Table 5 we record the optimal evaporation rate (the top number) and an average thinning rate (the bottom number) for the PDE fit and each of the five ODE fits. The full thickness-TBU instance is identified by the Trial and FT-TBU ID columns. The average thinning rate is either taken starting $2 \mathrm{~s}$ into the trial, or if the trial is $2 \mathrm{~s}$ or less, the value at the final time is recorded. The values corresponding to the best fit by an ODE model as determined by the smallest residual are shaded. In all instances, the overall thinning rate of the best ODE fit is larger than that of the PDE. This may reflect the tendency of the theoretical PDE FL intensity to lag the experimental FL intensity in later times. In most instances, the best fit overall thinning rate is larger than the evaporation rate, indicative of outward thinning that supports the notion that the Marangoni 
effect contributed to the thinning. Notably, for the S9v2t5 4:30 spot, which was categorized as evaporation-dominated in Luke et al., ${ }^{20}$ the evaporation rate is larger than the thinning rate, suggesting inward capillary tangential flow combats the thinning. Some short trials exhibit rapid dynamics which occur early on; the recorded thinning rate may not represent the entirety of the trial.

Table 5. The optimal evaporation rates are recorded along with estimates of average $\dot{h}^{\prime}$ for the mixedmechanism model fits (starting $2 \mathrm{~s}$ into the trial). All rates are measured in $\mu \mathrm{m} / \mathrm{min}$. PDE values are given by $v^{\prime}$ and $\partial_{t^{\prime}} h^{\prime}$; the rest are from the various ODE models. The value at the last time was used for trials less than $2 \mathrm{~s}$ in length. The five cases of ODE models are in order and denoted by subscripts on the evaporation value: $v_{E}^{\prime}, v_{O}^{\prime}, v_{F}^{\prime}, v_{D}^{\prime}$ and $v_{M}^{\prime}$. The shaded entries correspond to the model giving the best fit as determined by the smallest norm.

\begin{tabular}{|c|c|c|c|c|c|c|c|}
\hline Trial & $\begin{array}{c}\text { FT-TBU } \\
\text { ID }\end{array}$ & $\begin{array}{c}v^{\prime} \\
-\partial_{t^{\prime}} h^{\prime} \\
\end{array}$ & $\begin{array}{c}v_{E}^{\prime} \\
-\dot{h}^{\prime} \\
\end{array}$ & $\begin{array}{r}v_{O}^{\prime} \\
-\dot{h}^{\prime} \\
\end{array}$ & $\begin{array}{l}\boldsymbol{v}_{\boldsymbol{F}}^{\prime} \\
-\dot{\boldsymbol{h}}^{\prime}\end{array}$ & $\begin{array}{c}v_{D}^{\prime} \\
-\dot{h}^{\prime} \\
\end{array}$ & $\begin{array}{l}\boldsymbol{v}_{M}^{\prime} \\
-\dot{\boldsymbol{h}}^{\prime} \\
\end{array}$ \\
\hline \multirow{2}{*}{ S9v1t4 } & \multirow{2}{*}{$4: 00-$} & -6.26 & -28.7 & -30.2 & -13.5 & -16.4 & -24.1 \\
\hline & & -15.8 & -28.7 & -26.4 & -26.3 & -26.0 & -24.0 \\
\hline \multirow{2}{*}{ S9v2t1 } & \multirow{2}{*}{$3: 00-$} & -30.3 & -30.4 & -31.9 & -29.1 & -38.6 & -27.3 \\
\hline & & -21.2 & -30.4 & -28.5 & -29.2 & -20.6 & -38.1 \\
\hline \multirow{2}{*}{ S9v2t5 } & \multirow{2}{*}{$4: 00 \circ$} & -26.2 & -22.2 & -23.4 & -27.7 & -37.5 & -22.4 \\
\hline & & -18.2 & -22.2 & -20.1 & -20.2 & -25.1 & -30.0 \\
\hline \multirow{2}{*}{ S9v2t5 } & \multirow{2}{*}{$4: 30 \circ$} & -36.9 & -42.8 & -44.6 & -50.0 & -48.7 & -50.9 \\
\hline & & -23.4 & -42.8 & -35.7 & -36.5 & -38.2 & -44.9 \\
\hline \multirow{2}{*}{ S10v1t6 } & \multirow{2}{*}{$12: 30 \circ$} & -5.92 & -120 & -122 & 0 & -1.27 & -4.91 \\
\hline & & -7.60 & -120 & -0.0131 & -9.92 & -10.4 & -11.5 \\
\hline \multirow{2}{*}{$\mathrm{S} 13 \mathrm{v} 2 \mathrm{t} 10$} & \multirow{2}{*}{$6: 30-$} & -13.6 & -37.1 & -38.9 & -21.5 & -26.4 & -20.4 \\
\hline & & -21.8 & -37.1 & -33.6 & -31.7 & -31.1 & -30.8 \\
\hline \multirow{2}{*}{ S18v2t4 } & \multirow{2}{*}{$7: 30 \circ$} & -13.1 & -37.1 & -39.0 & -0.0011 & -25.2 & -20.3 \\
\hline & & -16.3 & -37.1 & -32.4 & -18.9 & 21.0 & -20.5 \\
\hline \multirow{2}{*}{ S27v2t2 } & \multirow{2}{*}{$5: 00-$} & -6.11 & -31.1 & -32.0 & -43.6 & -47.8 & -9.32 \\
\hline & & -16.0 & -31.1 & -28.6 & -23.9 & -38.8 & -30.9 \\
\hline
\end{tabular}

We plot $\partial_{r^{\prime}} \bar{u}^{\prime}$ or $\partial_{x^{\prime}} \bar{u}^{\prime}$ for the three examples from Section 3.3 in Figure 9 along with $\partial_{x^{\prime}} u^{\prime}$ from the relevant ODE models. In Figure 9b,c we also plot the evaporationonly PDE profiles. At least one ODE model does a decent job approximating the qualitative behavior of the PDE tangential flow profile after the first quarter second or so. A notable exception is the constant extensional tangential flow option for the S10v1t6 12:30 spot; this suggests the tangential flow profile is highly time-dependent. An average value for each model and instance is taken over the whole trial and recorded in Table 6.

In Table 6 we record the tangential flow profile data for each instance. The shaded entries correspond to the best fit. From left to right, the parameters correspond to the coefficients of the tangential flow terms from the case F, D, and M models, respectively. For most cases, the optimal tangential flow directions match the PDE results, 


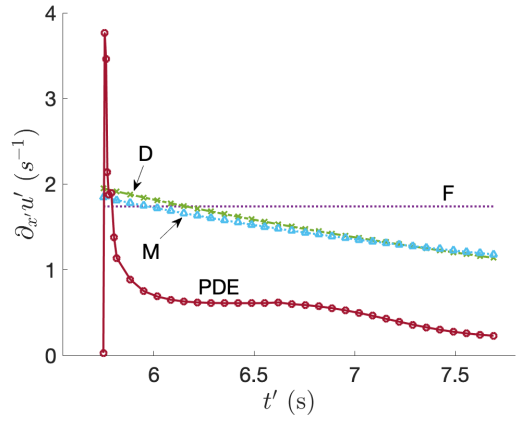

(a) S10v1t6 12:30 spot



(b) S27v2t2 5:00 streak

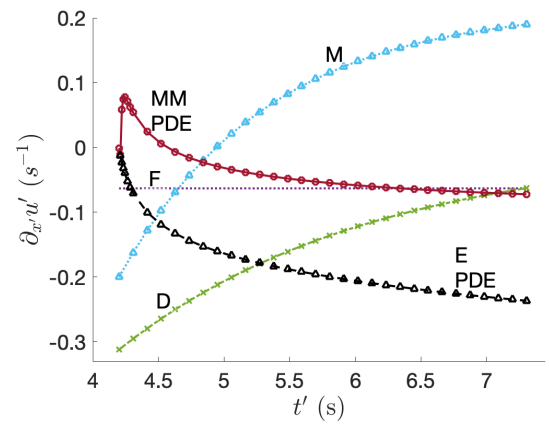

(c) S9v2t5 4:00 spot

Fig. 9. The mixed-mechanism $\partial_{r^{\prime}} \bar{u}^{\prime}$ or $\partial_{x^{\prime}} \bar{u}^{\prime}$ are plotted against the strain rate $\partial_{x^{\prime}} u^{\prime}$ for each ODE model with tangential flow.

an indicator that they were correctly classified in Luke et $a l .^{20}$ The three instances classified as evaporation-dominated or transitional thinning in Luke et al. ${ }^{20}$ show some amount of inward tangential flow in both the PDE and ODE best fits, which is consistent since evaporation-dominated thinning is characterized by inward tangential flow. In the cases of the S9v1t4 4:00 streak, S10v1t6 12:30 spot, S13v2t10 6:30 streak, and S18v2t4 7:30 spot, the ODE strain rates are always positive, which match the signs of the average strain rates of their corresponding PDE fits. The positive strain rate corresponds to outward tangential flow and so the thinning is likely influenced by the Marangoni effect. In the case of the S27v2t2 5:00 streak, the final strain rate signs of the best fit ODE and mixed-mechanism PDE models do not match. In this instance, the evaporation-only and mixed-mechanism PDEs show qualitative differences, and the evaporation-only PDE gives a better fit to the central data than the mixed-mechanism version as determined by the smaller norm of the difference of the experimental and theoretical FL intensities. This suggests that evaporation alone better explains the thinning, rather than a combination of evaporation and Marangoni flow. Perhaps 
Table 6. Estimates of the average extensional rate $\dot{\gamma}^{\prime}$, which is either $\partial_{r^{\prime}} \bar{u}^{\prime}$ for spots or $\partial_{x^{\prime}} \bar{u}^{\prime}$ for streaks, at the origin taken over the entire trial length in $\left(s^{-1}\right)$ for the mixed-mechanism model fits. These are compared with the optimal values from the three ODE models with tangential flow. The shaded entries correspond to the model giving the best fit as determined by the smallest norm.

\begin{tabular}{|l|l|l|l|l|l|l|l|l|}
\hline Trial & $\begin{array}{c}\text { FT-TBU } \\
\text { ID }\end{array}$ & $\dot{\gamma}^{\prime}$ & $\boldsymbol{a}_{\boldsymbol{F}}^{\prime}$ & $\boldsymbol{b}_{\mathbf{1} \boldsymbol{D}}^{\prime}$ & $\boldsymbol{b}_{\mathbf{2} \boldsymbol{D}}^{\prime}$ & $\boldsymbol{a}_{\boldsymbol{M}}^{\prime}$ & $\boldsymbol{b}_{\mathbf{1} \boldsymbol{M}}^{\prime}$ & $\boldsymbol{b}_{\mathbf{2} \boldsymbol{M}}^{\prime}$ \\
\hline S9v1t4 & $4: 00-$ & .150 & .189 & .164 & .0421 & .0316 & .418 & 5.75 \\
\hline S9v2t1 & $3: 00-$ & -.0218 & .0199 & -.0894 & .385 & .461 & -.490 & .0715 \\
\hline S9v2t5 & $4: 00 \circ$ & -.0427 & -.0631 & -.312 & .517 & .217 & -.417 & .882 \\
\hline S9v2t5 & $4: 30 \circ$ & -.0786 & -.0757 & -.963 & .708 & .360 & -.564 & .367 \\
\hline S10v1t6 & $12: 30 \circ$ & .572 & 1.74 & 1.95 & .277 & .656 & 1.19 & .423 \\
\hline S13v2t10 & $6: 30-$ & .147 & .172 & .138 & .102 & .173 & .0257 & .812 \\
\hline S18v2t4 & $7: 30 \circ$ & .172 & .674 & 2.41 & 8.85 & .0733 & 3.69 & 12.8 \\
\hline S27v2t2 & $5: 00-$ & .343 & -.201 & -.282 & .0761 & .714 & -.368 & .540 \\
\hline
\end{tabular}

the breakup dynamics of the streak in fact includes inward tangential flow. For the S27v2t2 5:00 streak, this theory is supported by the text in Section 3.3.

In general, the ODE models do a good job of capturing the essence of the dynamics of the PDE model fits. Each instance that we expect to have some outward tangential flow has at least one positive tangential flow parameter, and vice versa for the inward tangential flow instances (see Table 6). The PDE models are fit to more total data points since they consider the data across the breakup region and throughout the trial. As such, any single data point is less important in determining the optimal parameters that create the fit. Conversely, the ODE models are fit only to data taken from one spatial location (the center of breakup, corresponding to the origin of the PDE fits), and so significantly less data points are considered. As a result, the fit is more sensitive to a single data point. For these reasons, the PDE and ODE results must be compared with this caveat in mind: by subsetting the PDE results to the origin, we do not show most of the spatial data that influenced the choice of optimal PDE parameters, and so the PDE fit may not follow the data at the origin as well as the ODE fit. The PDE fits often lag behind the experimental data in later times; this is reflected in the slower (in general) average thinning rate $\partial_{t^{\prime}} h^{\prime}$ as compared to the ODE values $\dot{h}^{\prime}$ (see Table 5). However, the ODE data and fit is a simplification of the overall breakup dynamics, and viewing temporospatial data has value on its own.

Figure 10 compares the PDE and ODE best fit evaporation and thinning rate results to experimental point measurements reported in Nichols et al. ${ }^{39}$ For both histograms, a bin size of $5 \mu \mathrm{m} / \mathrm{min}$ was used. While the ODE rates show a wider range than the PDE results, there is significant overlap. The overall thinning rate is more comparable to the Nichols et al. ${ }^{39}$ data since that study could not separate evaporation from the other mechanisms affecting TF thickness. We expect our thinning rates to be larger than the point measurements since the Nichols et $a .^{39}$ study did not target breakup. While some of the ODE data lie outside the experimental distribution, many ODE thinning rate values are comparable, suggesting these simplified models 
return physically relevant quantities that cannot be otherwise estimated.

In Figure 11a we compare the maximum osmolarity values of the PDE and best fit ODE models. A bin size of $50 \mathrm{mOsmol} / \mathrm{L}$ was used. Both PDE and ODE peak osmolarity estimates are reasonable compared to other experimental and theoretical values. ${ }^{17,36}$ The ODE results show greater variation and exhibit larger maximal values on average. Peng et $a l .{ }^{42}$ and Braun et al. ${ }^{18}$ showed that diffusion reduces the peak osmolarity in TBU, which is only relevant for a model with spatial variation. As such, the lower PDE maximum osmolarity values are expected. Further, Braun et al. ${ }^{30}$ reported theoretical osmolarity values up to ten times the isotonic level, and so our largest osmolarity value, which is just over five times isotonic, is not unreasonable.

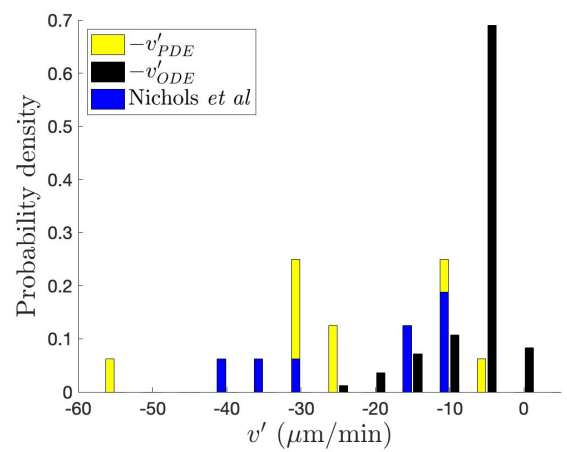

(a) Evaporation rates



(b) Overall thinning rates

Fig. 10. Histograms of rates of change plotted against experimental point measurements from Nichols et al.; ${ }^{39}$ note that the experiment cannot distinguish between $v^{\prime}$ and $\dot{h}^{\prime}$. The best fit ODE model data is shown as determined by the smallest norm. In (a) we have negated our evaporation rates to compare with the Nichols et al. ${ }^{39}$ data.

Figure $11 \mathrm{~b}$ records the minimum thicknesses of the PDE and best ODE fits. A bin size of $0.2 \mu \mathrm{m}$ was used. There is significant overlap of the PDE and ODE model results, suggesting that the simplified version can capture the end behavior of TF dynamics with a sufficient level of accuracy. The minimum TF thickness values are larger on average for the PDE models; this may be explained by the lag of the theoretical FL intensity behind the experimental data at later times in many of the PDE fits. ${ }^{20,25}$

Overall, there is more variability in the ODE results than the PDE results. We may overfit the subtleties of the dynamics with four parameters in the case M model, especially when the few data points of the central dynamics are essentially linear. Further, in some instances, the dynamics of the case $\mathrm{D}$ and $\mathrm{M}$ models are nearly indistinguishable, suggesting the additional parameter in the $\mathrm{M}$ model that mimics steady, outward tangential flow may not be necessary. The PDE data, which combines spatial and temporal information, is only fit with three parameters, reducing the likelihood of overfitting. Slight differences in the experimental data that are likely due to noise 


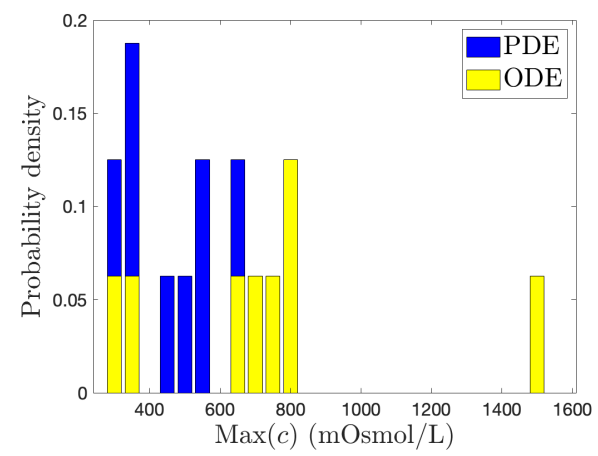

(a) Maximum osmolarity

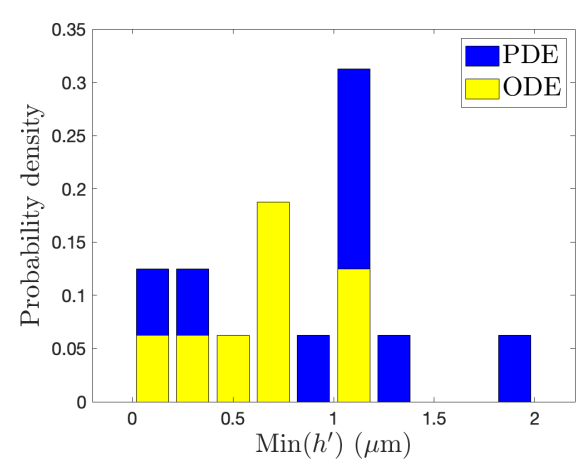

(b) Minimum TF thickness

Fig. 11. Histograms of maximum osmolarity and minimum TF thickness (final times of fit).

can affect the optimal parameters. Better time resolution would help get rid of the influence of outlier time levels on the optimization.

In order to compare with the PDE results, we scale with a characteristic length and horizontal velocity whose meanings are less clear in the context of the ODE model. The time scale we use is more of a characteristic time to bend the curve rather than the time to an overall decrease in FL intensity. We show the PDE results only for comparison; this ODE fitting process can be used on many other instances.

Our work is a basic science attempt to understand the driving causes of TBU and has important clinical implications. Our models are designed to capture mechanisms of TBU in the simplest way possible. The models provide a possibility of identifying the underlying mechanisms by fitting FL image data and can estimate the worst-case osmolarity in breakup regions (see Fig. 11a). Tear film breakup time (TBUT) and final osmolarity values strongly depend on the mechanisms of TBU. The ability of our models to separate the effects of Marangoni flow and evaporation is important because the first drives shorter TBUTs and the latter induces a higher final osmolarity value within the breakup region. The models tend to overestimate the osmolarity as compared to more complicated models. ${ }^{18,22}$ Our conclusions fall in line with previous osmolarity estimates ${ }^{17,20,36}$ and can be several times the isotonic value at the end of a trial. The instances for which Marangoni flow was important, as designated by positive tangential flow parameters in Table 4, do not exhibit a large increase in osmolarity. However, these instances are typically faster, and a subject with rapid TBU is more likely to be diagnosed with DED. ${ }^{54}$ In contrast, evaporation-driven instances, typically indicated by a higher evaporation rate and negative tangential flow parameters in Table 4, have higher final osmolarity values. Final TF thickness values can be estimated and are lower than PDE models on average, ${ }^{20}$ which helps explain the higher osmolarity values due to the inverse relationship between TF thickness and osmolarity (see Fig. 4a,c). These breakup parameter estimates and mechanistic iden- 
tifications should be of interest to clinicians and the fitting procedure could aid in the diagnosis of DED.

\section{Conclusions and future perspectives}

We have successfully determined whether evaporation, tangential flow, or a combination of the two cause TBU in a variety of instances by the relatively quick process of our ODE fitting procedure, and estimate breakup parameters that cannot currently be measured directly in vivo. In this paper, we validate our ODE fitting procedure by fitting the same data as in Luke et al. ${ }^{20}$ with simplified models and find good qualitative agreement of PDE ${ }^{20}$ and ODE results in most instances.

We are working on a machine learning approach to automatically identify breakup instances and fit the central data with our ODE models. This strategy could be applied on a large scale to obtain statistical information about a wide range of TBU shapes.

\section{Declarations}

\section{Ethics approval and consent to participate}

Approval was received from the Biomedical Institutional Review Board of Indiana University, Declaration of Helsinki principles were followed during data collection, and informed consent was obtained from all subjects.

\section{Competing interests}

None to declare.

\section{Funding}

This work was supported by National Science Foundation grant DMS 1909846. The content is solely the responsibility of the authors and does not necessarily represent the official views of the funding sources.

\section{Acknowledgements}

None to declare.

\section{References}

1. Norn M. Desiccation of the Precorneal Film: I. Corneal Wetting-Time. Acta Ophthalmol, 1969;47(4): 865880.

2. Lemp MA et al. The definition and classification of dry eye disease: Report of the Definition and Classification Subcommittee of the International Dry Eye WorkShop. Ocul Surf, 2007;5, 75-92.

3. Willcox MDP, Argüeso P, Georgiev GA, Holopainen JM, Laurie GW, Millar TJ, et al. The TFOS DEWS II Tear Film Report. Ocul Surf, 2017;15, 369-406. 
4. King-Smith PE, Begley CG, Braun RJ. Mechanisms, imaging and structure of tear film breakup. Ocul Surf, 2018;16, 4-30.

5. King-Smith PE, Hinel EA, Nichols JJ. Application of a novel interferometric method to investigate the relation between lipid layer thickness and tear film thinning. Invest Ophthalmol Vis Sci, 2010;51(5): 2418-2423.

6. Zhong L, Braun RJ, Begley CG, King-Smith PE. Dynamics of fluorescent imaging for rapid tear thinning. Bull Math Biol, 2019;81(1): 39-80.

7. Yokoi N, Georgiev GA. Tear-film-oriented diagnosis and therapy for dry eye. Dry eye syndrome: basic and clinical perspectives. Ed. by N Yokoi. London: Future Medicine, 2013; 96-108.

8. Yokoi N, Georgiev GA. Tear-film-oriented diagnosis for dry eye. Jpn J Ophthalmol, 2019;63, 127-136.

9. Berger R, Corrsin S. A surface tension gradient mechanism for driving the pre-corneal tear film after a blink. J Biomech, 1974;7, 225-38.

10. Craster RV, Matar OK. Dynamics and stability of thin liquid films. Rev Mod Phys, 2009;81(3): 1131 .

11. Sharma A, Ruckenstein E. Mechanism of Tear Film Rupture and Formation of Dry Spots on Cornea. J Coll Interface Sci, 1985;106, 12-27.

12. Sharma A, Khanna R, Ruckenstein E. A thin film analog of the corneal mucus layer of the tear film: an enigmatic long range non-classical DLVO interaction in the breakup of thin polymer films. Coll Surf B: Biointerfaces, 1999;14, 223-235.

13. Zhang L, Matar OK, Craster RV. Analysis of tear film rupture: Effect of non-Newtonian rheology. J Coll Interface Sci, 2003;262, 130-48.

14. Begley CG, Simpson T, Liu H, Salvo E, Wu Z, Bradley A, et al. Quantative Analysis of Tear Film Fluorescence and Discomfort During Tear Film Instability and Thinning. Invest Ophthalmol Vis Sci, 2013;54, 2645-2653.

15. Begley CG, Himebaugh N, Renner D, Liu H, Chalmers R, Simpson T, et al. Tear breakup dynamics: a technique for quantifying tear film instability. Optom Vis Sci, 2006;83(1): 15-21.

16. King-Smith PE, Reuter KS, Braun RJ, Nichols JJ, Nichols KK. Tear film breakup and structure studied by simultaneous video recording of fluorescence and tear film lipid layer images. Invest Ophthalmol Vis Sci, 2013;54(7): 4900-4909.

17. Peng CC, Cerretani C, Braun RJ, Radke CJ. Evaporation-driven instability of the precorneal tear film. Adv Coll Interface Sci, 2014;206, 250-264.

18. Braun RJ, Driscoll TA, Begley CG, King-Smith PE, Siddique JI. On tear film breakup (TBU): dynamics and imaging. Math Med Biol, 2018;35(2): 145-180.

19. Zhong L, Ketelaar CF, Braun RJ, Begley CG, King-Smith PE. Mathematical modelling of glob-driven tear film breakup. Math Med Biol, 2018;36(1): 55-91.

20. Luke RA, Braun RJ, Driscoll TA, Awisi-Gyau D, Begley CG. Parameter Estimation for Mixed-Mechanism Tear Film Thinning. Bull Math Bio, 2021;83(56): Available from: https://doi.org/10.1007/s11538-02100871-x.

21. Braun RJ, Driscoll TA, Begley CG. Mathematical Models of the Tear Film. Ocular Fluid Dynamics. Springer, 2019; 387-432.

22. Braun RJ, Gewecke NR, Begley CG, King-Smith PE, Siddique JI. A model for tear film thinning with osmolarity and fluorescein. Invest Ophthalmol Vis Sci, 2014;55(2): 1133-1142.

23. Winter KN, Anderson DM, Braun RJ. A model for wetting and evaporation of a post-blink precorneal tear film. Math Med Biol, 2010;27, 211-225.

24. Braun RJ. Dynamics of the tear film. Annu Rev Fluid Mech, 2012;44, 267-297.

25. Luke RA, Braun RJ, Driscoll TA, Begley CG, Awisi-Gyau D. Parameter Estimation for Evaporation-Driven Tear Film Thinning. Bull Math Bio, 2020;82(6): Available from: https://doi.org/10.1007/s11538-02000745-8.

26. Gilbard JP, Farris RL, Santamaria J. Osmolarity of tear microvolumes in keratoconjunctivitis sicca. Arch Ophthalmol, 1978;96(4): 677-681. 
27. Craig JP, K NK, Nichols JJ, Caffery B, Dua HS, Akpek EK, et al. The TFOS DEWS II Definition and Classification Report. Ocul Surf, 2017;15, 276-283.

28. Tomlinson A, Doane M, McFadyen A. Inputs and Outputs of the Lacrimal System: Review of Production and Evaporative Loss. Ocul Surf, 2009;7(4): 186-198.

29. Stahl U, Willcox M, Stapleton F. Osmolality and tear film dynamics. Clin Exp Optom, 2012;95(1): 3-11.

30. Braun RJ, King-Smith PE, Begley CG, Li L, Gewecke NR. Dynamics and function of the tear film in relation to the blink cycle. Prog Retin Eye Res, 2015;45, 132-164.

31. Lemp MA, Bron AJ, Baudouin C, Castillo JMB del, Geffen D, Tauber J, et al. Tear osmolarity in the diagnosis and management of dry eye disease. Am J Ophthalmol, 2011;151(5): 792-798.

32. Tomlinson A, Khanal S, Ramaesh K, Diaper C, McFadyen A. Tear film osmolarity: determination of a referent for dry eye diagnosis. Invest Ophthalmol Vis Sci, 2006;47(10): 4309-4315.

33. Versura P, Profazio V, Campos E. Performance of tear osmolarity compared to previous diagnostic tests for dry eye diseases. Curr Eye Res, 2010;35(7): 553-564.

34. Sullivan BD, Whitmer D, Nichols KK, Tomlinson A, Foulks GN, Geerling G, et al. An objective approach to dry eye disease severity. Invest Ophthalmol Vis Sci, 2010;51(12): 6125-6130.

35. Dartt D, Willcox M. Complexity of the tear film: importance in homeostasis and dysfunction during disease. Exp Eye Res, 2013;117, 1-3.

36. Liu H, Begley C, Chen M, Bradley A, Bonanno J, McNamara NA, et al. A Link between Tear Instability and Hyperosmolarity in Dry Eye. Invest Ophthalmol Vis Sci, 2009;50, 3671-79.

37. Pflugfelder SC. Tear dysfunction and the cornea: LXVIII Edward Jackson memorial lecture. Am J Ophthalmol, 2011;152(6): 900-909.

38. Belmonte C, Acosta MC, Merayo-Lloves J, Gallar J. What causes eye pain? Curr Ophthalmol Rep, 2015;3(2): 111-121.

39. Nichols JJ, Mitchell GL, King-Smith PE. Thinning rate of the precorneal and prelens tear films. Invest Ophthalmol Vis Sci, 2005;46(7): 2353-2361.

40. Kimball SH, King-Smith PE, Nichols JJ. Evidence for the major contribution of evaporation to tear film thinning between blinks. Invest Ophthalmol Vis Sci, 2010;51(12): 6294-6297.

41. Hamano H, Hori M, Mitsunaga S. Measurement of evaporation rate of water from the precorneal tear film and contact lenses. Contacto, 1981;25(2): 7-15.

42. Peng CC, Cerretani C, Li Y, Bowers S, Shahsavarani S, Lin M, et al. Flow evaporimeter to assess evaporative resistance of human tear-film lipid layer. Ind Eng Chem Res, 2014;53(47): 18130-18139.

43. Dursch TJ, Li W, Taraz B, Lin MC, Radke CJ. Tear-film evaporation rate from simultaneous ocularsurface temperature and tear-breakup area. Optom Vis Sci, 2018;95(1): 5-12.

44. Awisi-Gyau D. Characterization of tear breakup and its sensory effects. PhD Thesis, Indiana University, 2020; 1-202.

45. Carlson NB, Kurtz D, Hines C. Clinical procedures for ocular examination. 3. New York: McGraw-Hill, 2004; $1-487$.

46. Webber WRS, Jones DP. Continuous Fluorophotometric Method Measuring Tear Turnover Rate in Humans and Analysis of Factors Affecting Accuracy. Med Biol Eng Comput, 1986;24, 386-392.

47. Mota M, Carvalho P, Ramalho J, Leite E. Spectrophotometric analysis of sodium fluorescein aqueous solutions. Determination of molar absorption coefficient. Int Ophthalmol, 1991;15(5): 321-326.

48. Tiffany JM. The viscosity of human tears. Int Ophthalmol, 1991;15(6): 371-376.

49. Nagyová B, Tiffany J. Components responsible for the surface tension of human tears. Curr Eye Res, 1999;19(1): 4-11.

50. Wu Z, Begley CG, Port N, Bradley A, Braun R, King-Smith E. The effects of increasing ocular surface stimulation on blinking and tear secretion. Invest Ophthalmol Vis Sci, 2015;56(8): 4211-4220.

51. Nocedal J, Wright S. Numerical optimization. Berlin: Springer Science \& Business Media, 2006; 1-597.

52. LeVeque RJ. Finite Difference Methods for Ordinary and Partial Differential Equations: Steady-State and Time-Dependent Problems. Philadelphia: SIAM, 2007; 1-339. 
53. Howell P. Models for thin viscous sheets. Euro J Appl Maths, 1996;7, 321-343.

54. Wolffsohn JS, Arita R, Chalmers R, Djalilian A, Dogru M, Dumbleton K, et al. The TFOS DEWS II Diagnostic Methodology Report. Ocul Surf, 2017;15, 544-579. 